Reprod. Nutr. Dévelop., 1986, 26 (1 B), 227-243.

\title{
Sémiologie biochimique du foie chez les ruminants
}

\author{
J. P. BRAUN $\left({ }^{*}\right), P . \operatorname{BÉZILLE}\left({ }^{* *}\right), A \cdot G \cdot \operatorname{RICO}\left({ }^{*}\right)$ \\ (*) Laboratoire associé INRA, Toxicologie biochimique et métabolique, ENV, 31076 Toulouse, \\ (**) Laboratoire de Pathologie du Bétail, ENV, 31076 Toulouse.
}

Summary. Biochemical semiology of the liver in ruminants.

Many different serum biochemical tests can help in the diagnosis of liver disturbances in ruminants. The best tests for hepato-cellular damage are the measurement of enzymes such as glutamate dehydrogenase, sorbitol dehydrogenase and, if available, arginase or ornithine carbamoyl transferase. Disturbances of biliary function can be investigated through the measurement of so-called "cholestasis enzyme markers " such as gammaglutamyl transferase or alkaline phosphatases ; bilirubin and bile salts can also be helpful. Liver insufficiency can be approached through the measurement of serum albumin, fibrinogen and coagulation tests whereas inflammative and inductive processes are difficult to investigate. Moreover, liver clearances (bromosulfonephtalein or indocyanine green) can provide useful data about whole liver function.

Chez les animaux domestiques, les ruminants en particulier, comme chez I'homme, après l'examen clinique, l'exploration des troubles hépatiques peut mettre en jeu, un très grand nombre de moyens paracliniques : imagerie radiologique, échographique ou scintigraphique, examens histologiques de ponctions-biopsies et biochimie clinique.

La présente revue ne pouvant pas être exhaustive, un choix, parfois subjectif, a donc du être effectué parmi les nombreuses références disponibles. Cette revue est limitée aux principes d'utilisation et à quelques exemples d'exploitation de la biochimie clinique en sémiologie hépatique chez les ruminants, en ne se limitant cependant pas aux seuls examens facilement utilisables dans la pratique vétérinaire quotidienne.

\section{Principes généraux de l'exploration biochimique du foie.}

L'exploration des fonctions d'un organe ne peut être entreprise que dans la mesure ou l'on a une connaissance suffisante de leur support anatomique et de leurs mécanismes intimes. Les revues et ouvrages généraux sont nombreux en ce domaine (en particulier, Ashdown et Done, 1984 ; Darnis, 1969 ; Darnis et Polonovsky, 1983 ; Plaa, 1978). 
La biologie clinique se veut aussi peu invasive que possible : il en résulte qu'elle repose avant tout sur des dosages sanguins ou urinaires, exceptionnellement sur des analyses de tissus prélevés lors de biopsies (Herdt et al., 1983).

Lorsqu'un désordre hépatique quelconque survient, une série de phénomènes variés peuvent se superposer et il convient d'essayer de les aborder tous. Pour ce faire, on a l'habitude de distinguer assez arbitrairement (fig. 1) :

- des tests de cytolyse qui visent à évaluer les destructions ou au moins les souffrances cellulaires ;

- des tests de la fonction excréto-biliaire auxquels on adjoint, souvent, les tests de traversée que sont les clairances hépatiques biliaires, telle celle de la BSP (brome sulfone phtaléine) ;

- des tests d'insuffisance hépatique reflétant la plus ou moins grande perte des capacités métaboliques des hépatocytes ;

- des tests de la réaction inflammatoire hépatique ;

- des tests d'induction qui sont encore assez peu appliqués chez les animaux domestiques.

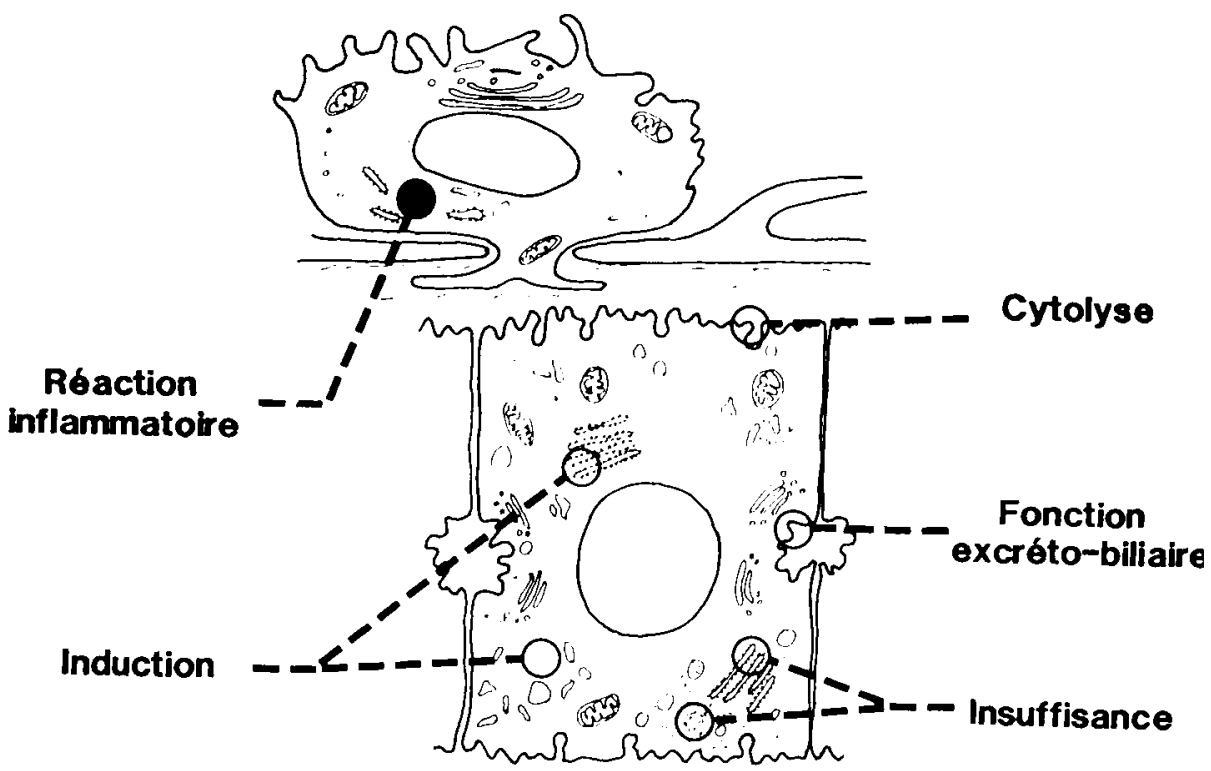

FIG. 1. - Représentation schématique des moyens de la sémiologie biochimique du foie.

Après l'examen systématique des principaux tests biochimiques et de leurs résultats, quelques situations caractéristiques seront présentées pour montrer comment les tests précédents se complètent en un "panorama " représentatif des désordres hépatiques. 


\section{Tests de cytolyse hépatique.}

\subsection{La cytolyse hépatique}

Le terme de cytolyse hépatique ne correspond à aucune définition anatomopathologique et recouvre tous les états de souffrance des hépatocytes. Ceux-ci vont des perturbations membranaires modérées qui peuvent intervenir lors d'une anoxie légère jusqu'à la nécrose cellulaire. L'étiologie de telles cytolyses peut-être très variable : affections bactériennes, virales, parasitaires, nutritionnelles ou toxiques. Ces phénomènes d'intensité très variable ont cependant un trait commun : l'augmentation de perméabilité de la membrane plasmique qui permet au contenu intracellulaire de s'échapper vers le milieu extracellulaire et, de là, en partie au moins vers le sang.

\subsection{Principe des tests de cytolyse : enzymologie}

En principe tous les constituants intra-cellulaires des hépatocytes peuvent être de bons marqueurs de cytolyse. En pratique, en biologie vétérinaire, on se limite à l'utilisation d'enzymes parmi lesquelles on sélectionne celles qui sont les plus spécifiques des hépatocytes.

Ce que I'on appelle improprement le « dosage des enzymes sériques ou plasmatiques " et qui est en fait la mesure de leurs concentrations d'activité catalytique permet, pour les enzymes d'origine hépatocytaire :

1) La détection de la lésion qui repose sur une augmentation nette de la concentration des enzymes dans le sang. Chaque jour, un certain pourcentage d'hépatocytes, variable d'un jour à l'autre, est renouvelé et leur contenu est déversé dans le milieu extra-cellulaire puis dans le sang. Cette quantité d'enzymes est assez peu variable d'un jour à l'autre pour un animal donné mais parfois très variable d'un animal à l'autre dans la même espèce : elle est à l'origine de la concentration, en général faible, d'enzymes d'origine hépatique (telles que la glutamate déshydrogénase ou l'ornithine carbamoyl transférase) présente dans le sang dans les conditions physiologiques. Cette concentration représente les valeurs de référence sériques pour les enzymes correspondantes. Par conséquent, toute augmentation importante de la concentration de ces enzymes témoigne d'une destruction cellulaire accrue ou d'une augmentation de la perméabilité des membranes des hépatocytes.

2) L'évaluation de l'étendue de la lésion qui est, en première approche, proportionnelle à l'augmentation d'activité mesurée. Cependant, il faut tenir compte ici de la demi-vie des enzymes dans le sang. En effet, ces protéines sont des molécules qui sont plus ou moins rapidement dégradées, captées selon des processus encore mal connus par différents organes. Elles ont donc des demi-vies très variables. On ne dispose que de peu de renseignements spécifiques aux ruminants ; chez ces derniers la demi-vie moyenne dans le plasma est voisine de 3 à $10 \mathrm{~h}$ (Collis, Symonds et Sansom, 1979 ; Anderson, Berrett et Patterson, 1978a). II en résulte donc que l'enzymologie ne permet de détecter que des souffrances 
cellulaires récentes. Si la mesure d'activité a lieu plusieurs heures ou plusieurs jours après que le processus lésionnel soit intervenu, les activités de certaines enzymes dont la demi-vie est courte peuvent avoir tellement diminué qu'elles peuvent paraître " normales ». Lorsqu'on soupçonne une telle situation, il convient alors de doser de préférence des enzymes dont la demi-vie est plus longue, par exemple, la glutamate déshydrogénase chez la vache (Collis, Symonds et Samson, 1979).

3) L'appréciation de la gravité de la lésion qui est souvent fondée sur le rapport activité des enzymes mitochondriales/activité des enzymes cytosolubles (Schmidt et Schmidt, 1979). On suppose, en effet, qu'une faible souffrance cellulaire qui ne se traduit que par une désorganisation de la membrane plasmique et non par la nécrose de la cellule, ne permet que le passage des constituants du cytosol vers le milieu extracellulaire et donc le sang. Par contre, une nécrose cellulaire permet à tous les constituants intracellulaires, et en particulier aux enzymes mitochondriales de s'échapper. Par conséquent, lorsqu'une lésion est assez peu intense, on assiste principalement à un accroissement d'activité des enzymes d'origine cytosolique et lorsque la souffrance cellulaire est très intense, les enzymes cytosoliques et mitochondriales sont toutes deux élevées. Ces considérations théoriques aident parfois à l'évaluation de la gravité de la lésion mais il faut bien garder en mémoire que toutes les cellules ne subissent pas un processus de la même intensité et que, par conséquent, ce n'est que la gravité moyenne de la souffrance tissulaire qui est ainsi approchée, d'autant plus que la demi-vie des enzymes peut, là encore, entraîner des distorsions.

4) L'évolution de la lésion qui est indiquée par celle de la concentration sanguine des enzymes. En effet, si on se rappelle que la durée de vie des enzymes est en général assez courte, il apparaît qu'une lésion ponctuelle se traduit très rapidement par le passage dans le sang d'une grande quantité d'enzyme puis, le processus lésionnel cessant, il n'y a plus d'enzymes déversées vers le sang. Par conséquent, la concentration plasmatique diminue rapidement, d'une manière approximativement exponentielle. Inversement, si la lésion cellulaire persiste ou s'intensifie, la concentration des enzymes dans le sang va en augmentant ou au minimum en se maintenant. Apparaît ainsi l'un des intérêts majeurs de l'enzymologie : pouvoir suivre l'évolution de la souffrance cellulaire. Mais cela impose de répéter fréquemment les mesures, à des intervalles de temps variables selon la $1 / 2$ vie de l'enzyme que l'on utilise comme marqueur de cytolyse.

\subsection{Les enzymes du foie chez les ruminants}

La distribution des enzymes dans les différents organes des animaux varie parfois de manière très notable en fonction de l'espèce et une enzyme hépatospécifique dans une espèce donnée ne l'est pas a priori dans une autre. Certaines enzymes sont très spécifiques des hépatocytes, en particulier I'OCT (ornithine carbamoyl transférase) (E.C. 2.1.3.3.) et l'arginase (E.C. 3.5.3.1) qui participent toutes deux au cycle de Krebs-Henseleit de synthèse de l'urée (Rasoloniraini, 1977 ; Garouachi, 1978). 
Quelques enzymes ont une très forte concentration dans le foie et également dans le rein. Elles peuvent, en général, être aussi utilisées comme " marqueurs " de cytolyse hépatique. En effet, lorsqu'une lésion du tubule rénal intervient, la majeure partie des enzymes est éliminée par voie urinaire et il est rare qu'une lésion rénale, même intense, soit accompagnée d'un accroissement d'activité enzymatique dans le plasma. Dans ce domaine, la glutamate déshydrogénase (GLD, E.C. 1.4.1.2) et la sorbitol déshydrogénase (SDH, E.C. 1.1.1.14) sont des enzymes utiles à l'exploration des cytolyses hépatiques.

Certaines enzymes qui ne sont pas spécifiques du foie, ont cependant d'assez fortes concentrations catalytiques dans le foie et leur activité sérique augmente donc lors de souffrance hépatique, même si leur signification diagnostique reste discutable. C'est, en particulier, le cas des transaminases (TGO = AST, E.C. 2.6.1.1 et TGP = ALT, E.C. 2.6.1.2) et de la lactate déshydrogénase (LDH, E.C. 1.1.1.27). Pour cette dernière, les isoenzymes permettent d'obtenir une meilleure spécificité puisque le foie renferme principalement les isoenzymes les plus rapides en électrophorèse (Garouachi, 1978 ; Rasoloniraini, 1977 ; Doxey, 1983).

\subsection{Valeurs usuelles et principales variations}

Chez les ruminants comme dans les autres espèces, la production de valeurs usuelles en enzymologie est considérablement gênée par l'absence de méthodes standardisées. Les mesures d'activité faisant appel à des techniques souvent très différentes, les résultats obtenus peuvent varier de manière très notable. Par conséquent, les valeurs données dans le tableau 1 n'ont qu'une simple valeur indicative.

Des élévations fortes et précoces des activités sériques ou plasmatiques de GLD, OCT, SDH, arginase, LDH et TGO ont été observées notamment dans les intoxications expérimentales ou naturelles par le tétrachlorure de carbone (Cornelius, 1961 ; Ford, 1967 ; Anwer et al., 1976 ; Mia et Koger, 1978), le cuivre (Gopinath et Howell, 1975), I'aflatoxine $B_{1}$ (Clark et al., 1984), les sporidesmines (Ford et Gopinath, 1976 ; Bézille et al., 1984), Phomopsis leptosromiformis (lupinose) (Allen, Moir et Macintosh, 1983 ; Malherbe et al., 1977), le séneçon (Ford, Ritchie et Thorpe, 1968), dans les infestations parasitaires par Theleria (Shlosberg et al., 1973), Fasciola hepatica (Roberts, 1968; Hughes, 1973 ; Barnouin, Mialot et Levieux, 1981), dans les abcès du foie (Shaw, 1974), ... Dans les conditions de la pratique, la fasciolose et les abcès du foie sont souvent inapparents au plan biologique.

\section{Tests de la fonction « excréto-biliaire ».}

\subsection{La fonction excréto-biliaire et principes de son exploration}

On entend, en général, sous ce vocable l'ensemble des processus qui concourent : 1) à capter des molécules circulant dans le sang ; 2) à leur faire subir des biotransformations variées qui visent le plus souvent à les rendre plus hydrosolubles ; 3) à les éliminer par voie biliaire vers le tube digestif. Ce mécanisme de 


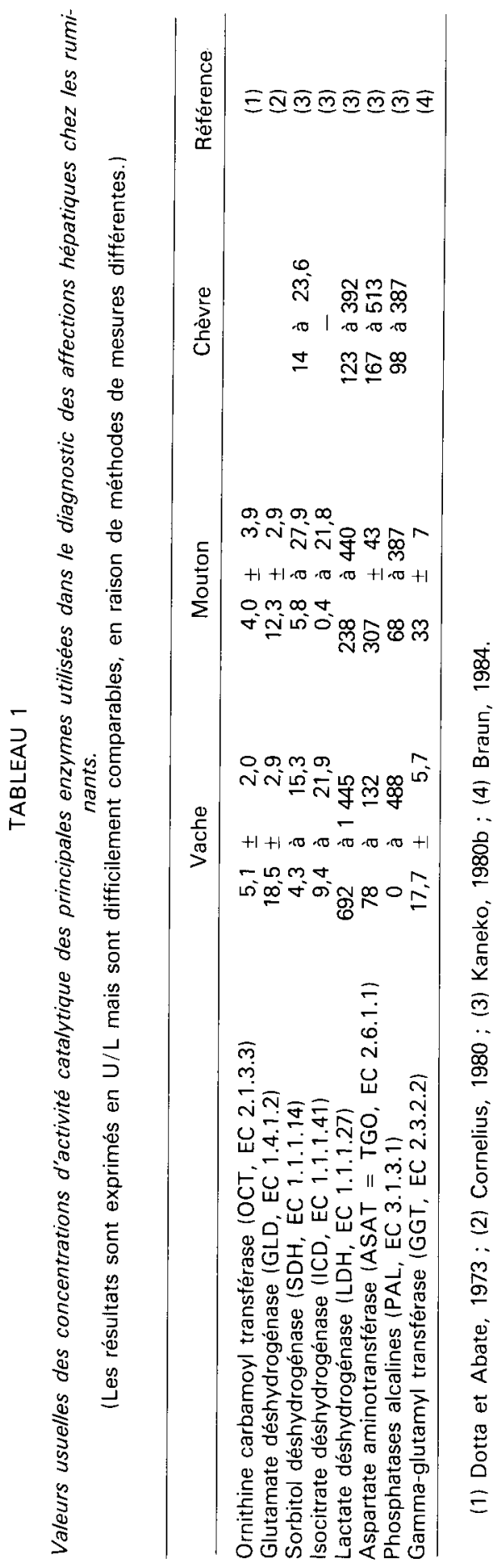


" capture-biotransformation-élimination » est subi par nombre de molécules endogènes : cholestérol et sels biliaires, pigments, hormones stéroïdes, etc... Il affecte également un grand nombre de xénobiotiques dont certains tels la BSP servent de traceurs pour évaluer son fonctionnement.

Deux grands types de moyens sont disponibles pour explorer cette fonction. Les premiers, les clairances hépatiques biliaires, explorent l'ensemble de la fonction mais ne permettent pas de préciser l'origine d'un trouble éventuel car elles dépendent du flux sanguin, de la capacité de capture hépatique, des fonctions des hépatocytes et des possibilités du drainage biliaire. Les seconds ne visent qu'à mettre en évidence un arrêt ou un ralentissement de l'écoulement biliaire, c'est-àdire une cholestase.

\subsection{Clairances hépatiques biliaires}

\subsubsection{Principes.}

- Clairance de la bromosulfonephtaléine (BSP) : la BSP est un colorant qui, après administration par injection intraveineuse rapide, est très rapidement capté par les cellules de Küpfer et transféré aux hépatocytes où il est conjugué avec le glutathion avant d'être éliminé dans la bile. Cependant le métabolisme de cette molécule n'est pas exclusivement hépatique : une fraction non négligeable est éliminée directement par le rein, 10 à $20 \%$ de la dose sont stockés par les muscles et la capacité de capture hépatique est saturable. II en résulte que ce n'est certainement pas le meilleur traceur pour une clairance biliaire. Cependant, c'est une méthode simple et peu coûteuse qui peut être intéressante, à condition de bien en connaître les limites.

- Clairance du vert d'indocyanine (ICG) : le principe est le même que pour la BSP mais cette molécule présente une beaucoup plus grande spécificité pour le foie (Sato, 1984). Sa clairance dépend de la dose administrée, laissant supposer que sa capacité de transport hépatique est également saturable. Son utilisation reste encore limitée, probablement en raison de son coût élevé et de son dosage qui nécessite des mesures dans le proche IR.

TABLEAU 2

Valeurs usuel/es des clairances hépatiques bilaires chez les ruminants.

\begin{tabular}{lccc} 
& $T 1 / 2(\min )$ & $k\left(\min ^{-1}\right)$ & Référence \\
\hline BSP : Vache lactation & & $0,16 / 0,24$ & $(1)$ \\
Vache hors lactation & $3,3 \pm 0,5$ & $0,22 \pm 0,03$ & $(1)$ \\
$\quad$ Vache 1 semaine avant vélage & & $0,151 \pm 0,007$ & $(2)$ \\
$\quad$ Vache le jour du vélage & & $0,146 \pm 0,007$ & $(2)$ \\
$\quad$ Vache 1 semaine après vélage & & $0,121 \pm 0,005$ & $(2)$ \\
Génisse & $4,5 \pm 0,3$ & $0,15 \pm 0,10$ & $(1)$ \\
$\quad$ Mouton & $2,0 \pm 0,3$ & $0,35 \pm 0,06$ & $(1)$ \\
$\quad$ Chèvre & $2,1 \pm 0,2$ & & \\
ICG : Vache & $2,8 \pm 0,5$ & $0,25 \pm 0,07$ & $(3)$ \\
$\quad$ Mouton & $4,8 \pm 0,5$ & & $(1)$ \\
\hline
\end{tabular}

T $1 / 2$ : demi-vie plasmatique ; $k$ : clairance fractionnelle.

(1) Cornelius, 1980 ; (2) Treacher, 1972 ; (3) Sen, Pahman et Mia, 1976 ; (4) Sato, 1984. 
3.2.2. Valeurs usuelles et principales variations pathologiques. - Chez les ruminants, la clairance de ces molécules est très rapide, comme on peut le voir dans le tableau 2. Par ailleurs, la clairance de la BSP est sensiblement ralentie par le jeûne (Cakala et Bienek, 1975).

Dans un très grand nombre d'affections hépatiques, la clairance de la BSP est ralentie : intoxication par $\mathrm{CCl}_{4}$ (Witte, 1970; Sen, Rahman et Mia, 1976), eczéma facial (Caple, Vandergraaff et Malmo, 1976 ; Bézille, Braun et Le Bars, 1984), fasciolose (Roberts, 1968 ; Witte, 1970)...

\subsection{Tests de cholestase}

3.3.1. Constituants explorés. - Dans ce domaine, deux groupes de constituants sont utilisés :

- des molécules qui sont éliminées par la bile dans les conditions physiologiques, comme le cholestérol, les sels et pigments biliaires ;

- des enzymes des pôles biliaires des hépatocytes et des épithéliums des voies biliaires : phosphatases alcalines (PAL, E.C. 3.1.3.1), gamma-glutamyl transférase (GGT, E.C. 2.3.2.2), 5'nucléotidase (5NU, E.C. 3.1.3.5), ... (Garouachi, 1978 ; Rasoloniraini, 1977).

3.2.2. Variations lors de cholestase. - Lors de stase biliaire, les divers composés éliminés par la bile tendent à refluer : leurs concentrations sanguines sont donc augmentées ; cela est particulièrement net pour la bilirubine, même si certains auteurs ont montré que son dosage n'était pas un test très sensible chez les bovins (Cornelius, 1980). De plus, I'hyperbilirubinémie entraîne une bilirubinurie. Un raisonnement semblable est probablement applicable aux sels biliaires mais ceux-ci n'ont été que très peu étudiés chez les ruminants (Anwer et al., 1976).

Dans ces conditions, l'augmentation de la pression dans les voies biliaires et l'action détergente des sels biliaires entraînent un décapage des enzymes des épithéliums " biliaires » ainsi qu'une induction de la synthèse de ces molécules. II en résulte une très forte augmentation de la concentration de ces enzymes dans le sérum ou le plasma. Cependant, les PAL ne seraient pas des indicateurs fiables de cholestase chez les ruminants, en raison de très fortes variations interindividuelles (Cornelius, 1980).

3.3.3. Valeurs usuelles et principales variations pathologiques. - Les valeurs usuelles des enzymes figurent dans le tableau 2, celles des substrats sont données dans le tableau 3.

Dans les cholestases expérimentales par ligature du canal cholédoque, on observe de fortes augmentations de la GGT (Ford, 1974 ; Ford et Gopinath, 1976 ; Grunder, 1977) ; il en est de même lors de fasciolose (Ben-Saïd, 1979 ; Sykes, Coop et Robinson, 1980 ; Sanderman et Howell, 1981) et d'eczéma facial (Towers et Stratton, 1978 ; Towers et Smith, 1978 ; Bézille, Braun et Le Bars, 1984). Par ailleurs, cette enzyme présente des augmentations modérées dans des affections où la stase ne paraît pas être le caractère dominant : carence en cobalt (Sutherland, Cordes et Carthew, 1979), ou cétose (Meissonnier et Rousseau, 1976). 
Des élévations de la bilirubine ont été, en particulier, relevées lors de lupinose (Allen, Moir et Macintosh, 1983), d'intoxication par $\mathrm{CCl}_{4}$ (Anwer et al., 1976), d'aflatoxicose B, (Clark et al., 1984), de babésiose (Akinboade, Diepolu, Adetunji, 1984) ou d'eczéma facial (Bézille, Braun, Le Bars, 1984). Des accroissements des sels biliaires ont été notés chez des moutons intoxiqués par $\mathrm{CCl}_{4}$ (Anwer et al., 1976).

\section{TABLEAU 3}

Valeurs usuelles des concentrations des principaux constituants plasmatiques utilisés pour le diagnostic des affections hépatiques des ruminants.

\begin{tabular}{|c|c|c|c|c|}
\hline & Vache & Mouton & Chèvre & Référence \\
\hline $\begin{array}{l}\text { Bilirubine }(\mathrm{mg} / \mathrm{l}) \\
\text { Cholestérol }(\mathrm{g} / \mathrm{l}) \\
\text { Sels biliaires }(\mu \mathrm{mol} / \mathrm{l}) \\
\text { Fibrinogène }(\mathrm{g} / \mathrm{l})\end{array}$ & $\begin{array}{c}1,0 \text { à } 4,7 \\
0,8 \text { à } 1,2 \\
54 \pm 4 \\
2 \text { à } 7\end{array}$ & $\begin{array}{l}1,0 \text { à } 4,2 \\
0,5 \text { à } 0,8 \\
48 \pm 3 \\
1,5 \text { à } 4\end{array}$ & $\begin{array}{l}0 \text { à } 1 \\
0,8 \text { à } 1,3\end{array}$ & $\begin{array}{l}(1) \\
(2) \\
(3) \\
(4)\end{array}$ \\
\hline $\begin{array}{l}\text { Protéines totales }(\mathbf{g} / \mathbf{l}) \\
\text { Albumine } \\
\text { alpha-1 Globulines } \\
\text { béta-1 Globulines } \\
\text { béta-2 Globulines } \\
\text { gamma-1 Globulines } \\
\text { gamma-2 Globulines }\end{array}$ & $\begin{array}{r}67,4 \text { à } 74,6 \\
30,3 \text { à } 35,5 \\
7,5 \text { à } 8,3 \\
8,0 \text { à } 11,2 \\
16,9 \text { à } 22,5\end{array}$ & $\begin{array}{r}60 \text { à } 79 \\
27 \text { à } 39 \\
3 \text { à } 6 \\
7 \text { à } 12 \\
4 \text { à̀ } 14 \\
7 \text { à } 22 \\
2 \text { à } 11\end{array}$ & 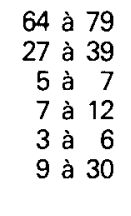 & (5) \\
\hline
\end{tabular}

(1) Cornelius, 1980 ; (2) Kaneko, $1980 \mathrm{~b}$; (3) Anwer et al., 1976 ; (4) Rushton, 1975 ; (5) Benja$\min , 1978$.

\section{Tests d'insuffisance hépato-cellulaire.}

\subsection{L'insuffisance hépato-cellulaire}

Elle se définit comme une diminution de l'aptitude métabolique moyenne des hépatocytes. Elle peut résulter soit d'un processus aigu rendant temporairement non fonctionnelle une partie de la masse hépatique, soit d'un processus chronique avec cirrhose ou fibrose dans lequel la masse hépatique fonctionnelle est réduite. II en résulte que les grandes fonctions métaboliques du foie sont diminuées. Notons cependant que les fonctions du foie peuvent être entièrement réalisées par seulement $20 \%$ de la masse hépatique : cela implique donc qu'une insuffisance n'apparaît que lorsque le parenchyme a subi des désordres massifs. Par ailleurs, la capacité de régénération du foie est telle que dans les processus aigüs il est rare que des signes biologiques d'insuffisance aient le temps de se manifester.

\subsection{Principes des tests d'exploration}

Lors d'insuffisance, les capacités de synthèse du foie sont diminuées. II en résulte que la concentration sanguine de nombre de protéines qui sont synthétisées par cet organe diminue. C'est, en particulier, le cas de l'albumine, du fibrinogène et de différents autres facteurs de la coagulation, des pseudocholinestéra- 
ses, etc. Notons cependant que les capacités de synthèse des protéines dépendent également de la couverture des besoins nutritionnels, que la concentration d'albumine peut être abaissée lors de néphropathie, d'entéropathie exsudative, de malabsorption, ou d'accroissements du catabolisme (par exemple pyélonéphrite, colibacillose, paratuberculose, ...) ... autant d'interférences qui compliquent l'interprétation des résultats. La demi-vie de ces différents constituants est très variable : il en résulte que la rapidité avec laquelle une insuffisance sera reflétée diffère notablement ; à cet égard, l'albumine, dont le taux de renouvellement quotidien est voisin de $5 \%$ chez les bovins (Cornelius, 1980), est un assez mauvais test alors que les facteurs de la coagulation réagissent de manière plus précoce.

Par ailleurs, les capacités métaboliques de l'organe peuvent être explorées en effectuant des clairances métaboliques, c'est-à-dire en mesurant l'aptitude du foie à capter et transformer une molécule endogène ou exogène administrée, en général, par voie veineuse. Lors d'insuffisance, ces clairances sont ralenties. Dans ce domaine assez peu exploité, on a utilisé la clairance du galactose, en particulier chez la chèvre (Treacher, 1972). Des travaux plus récents ont mis à profit la mesure du temps de demi-vie (T 1/2) de l'antipyrine, test plus habituellement employé pour juger d'une induction hépatique entraînant une diminution du $T 1 / 2$; dans le cas présent, l'insuffisance se manifeste par une augmentation du T 1/2 (Getachew, 1984). Dans le même ordre d'idées, nombre d'enzymes intervenant dans la biotransformation des xénobiotiques pourront peut-être servir également lorsque des études détaillées auront été effectuées : c'est en particulier le cas de l'éthoxycoumarine O-déméthylase, de l'aminopyrine $\mathrm{N}$-déméthylase, de la glucuronosyl transférase, etc. (Galtier et Larrieu, 1986).

Enfin lors d'insuffisance hépato-cellulaire, les différentes clairances biliaires sont très fortement ralenties (Cornelius, 1980).

\subsection{Valeurs usuelles et principales variations pathologiques}

Les valeurs usuelles des constituants utilisés pour explorer une insuffisance hépato-cellulaire sont données dans le tableau 3. II est impossible de fournir des valeurs usuelles des temps de coagulation qui dépendent trop notablement des méthodes employées.

Des diminutions de l'albumine sérique ont été observées, en particulier, lors d'infestations de ruminants par Schistosoma matthei (Van Zyl, 1974), Haemoncus contortus et Trichostrogylus colubriformis (Zajicek et al., 1972), Fasciola hepatica (Roberts, 1968 ; Zajicek et al., 1972 ; Girardi, Lafranchi et Abate, 1982), lors d'intoxication par le séneçon (Ford, Ritchie et Thorpe, 1968), lors de fibrose ou d'abcès étendus du foie (Barnouin, Mialot et Levieux, 1981). De même des diminutions des temps de coagulation ont été notées lors de fasciolose chez les bovins (Anderson et al., 1981).

\section{Tests de la réaction inflammatoire hépatique.}

\subsection{La réaction inflammatoire hépatique}

Elle intervient lors de la plupart des aggressions hépatiques. Souvent la congestion qu'elle entraîne se traduit par une faible cholestase transitoire. La réaction 
de l'organisme est manifestée par les mécanismes biochimiques locaux classiques de l'inflammation avec synthèse d'une variété de médiateurs chimiques et de protéines.

\subsection{Principes d'exploration}

En biologie clinique animale, l'exploration de la réaction inflammatoire hépatique est très limitée et ne fait pas appel, comme en biologie humaine, au dosage des "protéines de l'inflammation " (Alper, 1974; Bienvenu, 1984); de plus celles-ci ne sont guère spécifiques des réactions inflammatoires hépatiques; enfin, la signification des différentes fractions protéiques reste mal établie chez les animaux (Kaneko, 1980a) ; il en est de même de l'hypozincémie et de l'hypercuprémie qui accompagnent nombre de réactions inflammatoires (Renoult, 1985).

II en résulte qu'on est limité à l'exploitation de l'électrophorégramme et au calcul soit de la concentration des globulines totales, soit de celle des béta- ou des béta- + gamma-globulines, sans que les variations notées soient spécifiques du foie (Liberg, 1977b).

\subsection{Valeurs usuelles et principales variations pathologiques}

Le nombre des fractions protéiques et les valeurs usuelles correspondantes dépendent de la méthode d'électrophorèse utilisée : acétate de cellulose, gel d'amidon, etc. Les valeurs données dans le tableau 3 n'ont donc qu'une simple valeur indicative; des détails complémentaires peuvent être trouvés notamment dans des articles de Liberg (1977a) et de Lauerman et al. (1982). Par ailleurs, des méthodes plus grossières ont permis une évaluation des immunoglobulines par des tests de floculation (Pfeiffer et al., 1977; Sandholm, 1974) et des méthodes plus fines permettent la séparation des différentes classes d'immunoglobulines (Williams, Maxwell et Spooner, 1975).

Lors de réaction inflammatoire brève, les variations ne sont en général pas détectables. Par contre de fortes augmentations des globulines sont notées dans tous les processus d'évolution lente : aflatoxicose subaigüe (Miller et al., 1984), fasciolose et fibrose étendue (Barnouin, Mialot et Levieux, 1981).

\section{Tests de l'induction hépatique.}

Les inducteurs sont des molécules qui, in vivo, sont capables d'augmenter la synthèse hépatique d'un système enzymatique et/ou d'en réduire la vitesse de dégradation (Cinti, 1982). Il s'agit, en général, d'enzymes participant à la biotransformation des médicaments et les inducteurs sont très nombreux : outre certaines hormones, ce sont notamment le phénobarbital et d'autres xénobiotiques. Leur action se traduit par la synthèse d'une ou plusieurs molécules qui ne sont pas produites par le foie dans les conditions physiologiques ou par l'accroissement de synthèse de molécules qui sont déjà produites par cet organe. Ce processus prend place notamment dans le réticulum endoplasmique lisse et se manifeste par l'accroissement de l'activité de nombre d'enzymes " microsomales". 
L'induction n'a été que très peu étudiée chez les ruminants. Cependant, comme dans d'autres espèces il a été établi que le phénobarbital avait un effe: inducteur manifesté par une diminution du temps de sommeil induit (Cooper et Hawk, 1978) ou par un accroissement de l'activité de l'amidopyrine $\mathrm{N}$ déméthylase (Gopinath et Ford, 1976).

\section{Exemples de situations caractéristiques.}

Ainsi qu'il a été précédemment souligné, un désordre hépatique n'est que très rarement caractérisé par la perturbation d'un seul des processus envisagés cidessus. En règle générale, les diverses fonctions du foie sont affectées, souvent avec une intensité différente, souvent aussi à des moments différents de l'évolution de l'affection. Les situations décrites ci-après ne se veulent que des exemples et ne sont pas représentatives de la totalité de la pathologie hépatique des ruminants.

\subsection{Intoxication expérimentale par le tétrachlorure de carbone}

C'est l'un des très rares cas où, pour des doses modérées de $\mathrm{CCl}_{4}$, la cytolyse domine sans que les autres fonctions hépatiques soient perturbées de manière notable ou constante.

La cytolyse se manifeste par de très fortes et très précoces élévations des enzymes hépatocytaires (Boyd, 1962 ; Holtenius et Jacobson, 1966 ; Ford, 1965 ; Keller, 1974) qui reviennent rapidement à leurs valeurs usuelles: une semaine pour SDH, GLD, OCT et un peu plus pour TGO (Ford, 1967). Le plus souvent, les signes de stase liés à la réaction inflammatoire locale sont discrets ou absents (Boyd, 1962 ; Harvey et Hoe, 1971 ; Sen, Rahman et Mia, 1976) ; dans d'autres cas des perturbations notables de la bilirubine, des acides biliaires et de la GGT plasmatiques ont été rapportées (Ford, 1974 ; Anwer et al., 1976 ; Anderson et al., 1981). L'insuffisance hépato-cellulaire est modérée et transitoire et peut paraître sans effets sur l'albumine ou les facteurs de coagulation (Sen, Rahman et Mia, 1976) ou bien diminuer leur synthèse (Anderson et al., 1981).

\subsection{Eczéma facial. Intoxication par les Sporidesmines}

Les Sporidesmines, mycotoxines de Pythomyces chartarum, déterminent une très forte cholestase intra-hépatique dont témoignent des accroissements considérables de GGT, des PAL, de la bilirubine (Smith et al., 1975 ; Smith, Coe et Embling, 1978 ; Towers, 1978 ; Towers et Stratton, 1978 ; Bézille, Braun et Le Bars, 1984) accompagnés d'élévations du cholestérol (Done, Mortimer et Taylor, 1962 ; Leaver, 1968) et des acides bilaires (Peters et Mortimer, 1970) ; parallèlement la clairance de la BSP est fortement ralentie (Caple, Vandergraaf et Malmo, 1976 ; Bézille, Braun, et Le Bars, 1984). La cytolyse secondaire à la stase est reflétée par des augmentations massives de l'OCT, de la TGO, de la SDH, de la GLD (Marasas et al., 1972 ; Ford, 1974 ; Towers et Smith, 1978 ; Bézille, Braun, 
Le Bars, 1984). La réaction inflammatoire violente entraîne un accroissement des béta- et gamma-globulines (Mortimer, 1962 ; Leaver, 1968 ; Bézille, Braun et Le Bars, 1984). Quant à la fibrose progressive qui s'installe, elle est responsable de diminutions de la concentration de l'albumine (Mortimer, 1962).

\subsection{Fasciolose hépatique expérimentale}

Lors de fasciolose, la migration des larves dans le parenchyme hépatique produit une cytolyse qui reste le plus souvent modérée et qui est démontrée par des accroissements souvent durables mais peu intenses des activités plasmatiques de GLD, SDH, TGO (Hughes, Treacher et Harness, 1973, 1974 ; Anderson, Berrett et Patterson, 1978 ; Sykes, Coop et Robinson, 1980). Parallèlement, les troubles de cholestase sont à l'origine d'accroissements persistants de la GGT plasmatique (Knight, 1978 ; Anderson, Berrett et Patterson, 1978b ; Sirag et al., 1981). Lors d'évolution prolongée, une insuffisance hépato-cellulaire se manifeste par une diminution de l'albuminémie alors que la réaction inflammatoire détermine une augmentation des globulines (Roberts, 1968 ; Barnouin, Mialot et Levieux, 1981) ; simultanément, la clairance de la BSP est fortement ralentie (Roberts, 1968).

\section{Conclusion :}

Chez les ruminants, l'exploration biochimique du foie reste bien souvent partielle faute d'informations de base sur la valeur sémiologique de certains tests. Par ailleurs, elle ne peut prétendre fournir à elle seule la réponse à tous les problèmes de diagnostic en hépatologie que ce soit dans le cadre de la clinique quotidienne ou dans celui, plus exigeant, de la recherche. Ce n'est que l'un des éléments qui s'articulent avec l'examen clinique et les autres examens complémentaires. II n'en reste pas moins que par la simplicité de ses méthodes, la rapidité de ses réponses, la qualité de ses informations, la biochimie clinique reste encore irremplaçable dans l'exploration hépatique des ruminants.

Tères Journées sur la Nutrition et l'Alimentation des Herbivores, I.N.R.A., Paris, 21 et 22 mars 1985.

\section{Références}

AKINBOADE O. A., DIEPOLU O. O., ADETUNJI A., 1984. Enzyme and bilirubin reactions in bovine babesiosis. Ann. Rech. vét., 15, 313-318.

ALLEN J. G., MOIR R. J., MACINTOSH J. B., 1983. Ovine lupinosis resulting from the ingestion of lupin seed naturally infected with Phomopsis leptostroformis. Austr. vet. J., 60, 206-208.

ALPER C. A., 1974. Plasma proteins measurements as a diagnostic aid. New Engl. J. Med., 291,287290.

ANDERSON P. H, BERRETT S., PATTERSON D. S. P., 1978a. The rate of clearace of four liver-derived enzymes from the plasma of young cattle. Zbl. vet. Med., 28A, 808-811.

ANDERSON P. H., BERRETT S., PATTERSON D. S. P., 1978b. Resistance to Fasciola hepatica in cattle. II. Biochemical and morphological examination. J. comp. Path., 88, 245-251. 
ANDERSON P. H., MATTEWS J. G., BERRET S., BRUSH P. J., PATTERSON D. S. P., 1981. Changes in plasma enzyme activities and other blood components in response to acute and chronic liver damage in cattle. Res. vet. Sci, 31, 1-4.

ANWER M. S., ENGELKING L. R., GRONWALL R., KLENTZ R. D., 1976. Plasma bile acid elevation following $\mathrm{CCl}_{4}$ induced liver damage in dogs, sheep, calves and ponies. Res. vet. Sci., 20, 127-130.

ASHDOWN R. R., DONE S., 1984. Coulour atlas of veterinary anatomy. Baillière Tindall, London.

BARNOUIN J., MIALOT M., LEVIEUX D., 1981. Evaluation de la pathologie hépatique des bovins sur un prélèvement de sang. Relations avec l'histopathologie. Ann. Rech. vét,, 12, 363-369.

BENJAMIN M. M. 1978. Outline of veterinary clinical pathology. lowa State Univ. Press, Ames.

BEN SAID N. S., 1979. Fasciolose ovine: étude des variations de quelques paramètres hématologiques et biochimiques au cours d'infestations naturelles et après traitement par le Rafoxamide. Th. Doct. Méd. vét., Sidi Thabet.

BÉZILLE P., BRAUN J. P., LE BARS J., 1984. Première identification de l'eczéma facial chez les ovins en France. Aspects épidémiologiques, cliniques et biologiques. Rec. Méd. vét., 160 , 339-347.

BIENVENU J., 1984. Les protéines de la réaction inflammatoire. Définition, physiologie et méthodes de dosage. Ann. Biol. clin., 42, 47-52.

BLACKSHAW C., 1978. Serum gamma-glutamyltransferase in the diagnosis of liver disease in cattle. N.Z. Vet. J., 26, 25-26.

BOYD J. W., 1962. The comparative activity of some enzymes in sheep, cattle and rats. Normal serum and tissue levels and changes during experimental liver necrosis. Res. vet. Sci., 3, 256268.

BRAUN J. P., 1984. Contribution à l'étude de la distribution et de l'utilisation de la gammaglutamyltransférase chez les animaux. Th. Doct. Sci., Toulouse.

CAKALA S., BIENEK K., 1975. Bromosulphtalein clearance and total bilirubin level in cows deprived of food and water. Zbl. vet. Med., 22A, 605-610.

CAPLE I. W., VANDERGRAAFF R., MALMO J., 1976. Use of sulphobromophtalein liver function tests in cows with facial eczema. Austr. vet. J., 52, 192-193.

CINTI D.L., 1978. Agents activating the liver microsomal mixed function oxidase system. Pharmac. Ther. A, 2, 727-749.

CLARK J. D., HATCH R. C., MILLER D. M., JAIN A. V., 1984. Caprine aflatoxicosis : experimental disease and clinical pathological changes. Am. J. vet. Res., 45, 1132-1135.

COLLIS K. A., SYMONDS H. W., SANSOM B. F., 1979. The half-life of glutamate dehydrogenase in the plasma of dry and lactating cows. Res. vet. Sci, 27, 267-268.

COOPER B. S., HAWK H. W., 1978 . Effect of liver microsome enzyme-inducing drugs on blood serum pentobarbital clearance and sleep time in sheep and rabbits. Am. J. vet. Res., 39, 649-652.

CORNELIUS C. E., 1961. Serum isocitric dehydrogenase activities in domestic animals with experimental hepatic necrosis and in equine hepathopathy. Cornel/ Vet., 51, 559-568.

CORNELIUS C. E., 1980. Liver function, 201-257. In KANEKO J. J., Clinical biochemistry of domestic animals. Acad. Press, New York.

DARNIS F., 1969. Structures hépatiques. Encycl. Méd. Chir., 7005 A10.

DARNIS F., POLONOVSKY J., 1983. Grandes fonctions métaboliques du foie. Encycl. Méd. Chir., 7005 B10.

DONE J., MORTIMER P.H., TAYLOR A., 1962. The experimental intoxication of sheep with sporidesmin a metabolic product of Pithomyces chartarum. Res. vet. Sci., 3, 161-171.

DOTTA U., ABATE O., 1973. La determinazione della ornitina carbamil transferase nei bovini e nei cane. Nuova Vet., 49, 14-25.

DOXEY D. L., 1983. The value of isoenzymes in the diagnosis of disease in ruminants. Vet. Annu. 24, 112-117.

FORD E. J. H., 1967. Changes in the activity of ornithine carbamoyl transferase in the serum of cattle and sheep with hepatic lesions. J. comp. Path., 75, 299-308.

FORD E. J. H., 1974. Activity of sorbitol dehydrogenase in the serum of sheep and cattle with liver damage. J. comp. Pathol., 77, 405-411. 
FORD E. J. H., 1974. Activity of gamma-glutamyl transpeptidase and other enzymes in the serum of sheep with liver or kidney damage. J. comp. Path., 84, 231-243.

FORD E. J. H., GOPINATH C., 1976. The excretion of phylloerythrin and bilirubin by calves and sheep. Res. vet. Sci., 21, 12-18.

FORD E. J. H., LAWRENCE J. A., 1965. Hepatic and serum changes following repeated administration of small amounts of carbon tetrachloride to sheep. J. comp. Path., 75, 185202.

FORD E. J. H., RITCHIE H. E., THORPE E., 1968. Serum changes folowing the feeding of ragwort to calves. J. comp. Path., 78, 207-218.

GALTIER P., LARRIEU G, 1986. Enzymologie hépatique de biotransformation chez l'agneau. Description et évolution en cours de fasciolose. Reprod. Nutr. Dévelop., 26, 375-376.

GAROUACHI M., 1978. Enzymologie sérique chez les petits ruminants. Th. Doct. Vét. Toulouse.

GETACHEW W.M., 1984. Contribution à l'étude des perturbations hépatiques au cours de la fasciolose expérimentale de l'agneau par les tests à la BSP et à l'antipyrine. Maîtrise Sci. vét., Toulouse.

GIRARDI C., LAFRANCHI P., ABATE O., 1982. Fasciolose caprine : étude d'une grave infestation dans un élevage de la province de Turin. Point vét., 14, 49-56.

GOPINATH C., FORD E. J. H., 1976. The effect of induced hepatic microsomal amidopyrine demethylase activity on the susceptibility of the liver of the calf and horse to carbon disulphide. J. comp. Path., 86, 251-258.

GOPINATH C., HOWELL J. MCC., 1975. Experimental chronic copper toxicity in sheep. Changes that follow the cessation of dosing at the onset of hemolysis. Res. vet. Med., 19. 35-43.

GRUNDER H. D., 1977. La signification diagnostique de quelques enzymes sériques chez les bovins. Point vét. 6, 49-55.

HARVEY D. G., HOE C. M., 1971. The application of some liver function tests to sheep dosed with carbon tetrachloride and hexachlorophene. Vet. Rec. 88, 562-569.

HEALY P. J., 1968. Serum ornithine carbamyl transferase activity in sheep and cattle. Clin. chim. Acta, 22, 603-609.

HERDT T. H., GOEDERS L., LIESMAN J. S., EMERY R. S., 1983. Test for estimation of bovine lipid content. J. am. vet. med. Ass., 182, 953-955.

HOLTENIUS P., JACOBSON, S. O., 1966. Ornithine carbamyl transferase activity in ruminants. Cornell Vet., 56, 187-195.

HUGHES D. L., 1973. Plasma enzyme changes in goats infected with Fasciola hepatica and the effect of Nitroxinyl. Res, vet. Sci., 15, 249-255.

HUGHES D. L., TREACHER R. J., HARNESS E., 1973. Plasma enzyme changes in goats infected with Fasciola hepatica and the effect of Nitroxynil. Res. vet. Sci., 15, 249-255.

HUGHES D. L., TREACHER R. J., HARNESS E., 1974. The anthelmintic activity of Diamphenethide against immature Fasciola hepatica in goats and the course of experimental infections demonstrated by plasma enzyme changes. Res. vet. Sci. 17, 302-311.

JACOBS R. M., VALLI V. E. O., WILKIE B. N., 1980. Serum electrophoresis and immunoglobulin concentration in cows with Iymphoma. Am. J. vet. Res., 41, 1942-1946.

KANEKO J. J., 1980a. Serum proteins and the dysproteinemias, 97-118. In KANEKO J. J., Clinical biochemistry of domestic animals, Acad. Press, New York.

KANEKO J. J., 1980b. Clinical biochemistry of domestic animals. Acad. Press, New York.

KELLER P., 1974. Lactate dehydrogenase isoenzymes in normal bovine serum and during experimental liver and muscle damage. Res. vet. Sci., 17, 49-58.

KNIGHT R. A., 1978. Experimental cross infections of Fasciola hepatica in lambs and calves. J. Parasitol., 64, 601-605.

LAUERMAN L. H., RUPPANNER R., NORMAN B. B., ADAMS C. J., FARVER T. B., 1982. Metabolic and cellular profile testing in calves maintained under feedlot conditions : protein fractions and lactate dehydrogenase isoenzymes. Change over time. Am. J. vet. Res., 43. 884-886.

LEAVER D. D., 1968. Sporidesmin poisoning in the sheep. A comparison of some changes in clinical signs and serum constituents following sporidesmin poisoning and experimental obstruction of the common bile duct. Res. vet. Sci., 9, 265-273. 
LIBERG P., 1977a. Agarose gel electrophoretic fractionation of serum proteins in adult cattle. I. A study of clinically healthy cows. Acta vet. scand., 18, 40-53.

LIBERG P., 1977b. Agarose gel electrophoretic fractionation of serum proteins in adult cows. II. A study of cows with different diseases. Acta vet. scand., 18, 335-348.

MALHERBE W. D., KELLERMAN T. S., KRIEK N. P. J., HAUPT W. H., 1977. Gammaglutamyltransferase activity in sheep serum : normal values and an evaluation of its potential for detecting liver involvement in experimental lupinosis. Onderstepoort J. vet. Res., 44, 2938.

MARASAS W. P. O., ADELAAR T. F., KELleRMAN T. S., MinNe J. A., VAN RENSBURG I. B., BURROUGS G. W., 1972. First report of facial eczema in sheep in South Africa. Onderstepoort J. vet. Res., 29, 107-112.

MEISSONNIER E., ROUSSEAU P., 1976. Les tests enzymatiques dans l'exploration fonctionnelle du foie chez les bovins, 633-340. In MEISSONNIER E., Rapports et résumés, $9^{\circ}$ Congr. int. sur les maladies du bétail, ITCF, Maisse.

MIA A. S., KOGER H. D., 1978. Direct colorimetric determination of serum arginase in various domestic animals. Am. J. vet. Res., 39, 1381-1383.

MILLER D.M., CLARK J. D., HATCH R. C., JAIN A. V., 1984. Caprine aflatoxicosis : serum electrophoresis and pathologic changes. J. am. vet. med. Ass., 45, 1136-1141.

MORTIMER P. H., 1962. The experimental intoxication of sheep with sporidesmin, a metabolic product of Pithomyces chartarum. III. Some changes in cellular components and coagulation properties of the blood, in serum proteins and in liver function. Res. vet. Sci, 3, 269-286.

PETERS J. A., MORTIMER P. H., 1970. Sporidesmin poisoning in sheep. The effects on lipid and bile acid metabolism as reflected in serum. Res. vet. Sci, 11, 183-188.

PFEIFFER N. E., McGUIRE T. C., BENDEL R. B., WEIKEL J. M., 1977. Quantitation of bovine immunoglobulins : comparison of single radial immunodiffusion, zinc sulfate turbidity, serum electrophoresis and refractometer method. Am. J. vet. Res., 38, 693-698.

PLAA G. L., 1978. Evoling concepts in drug-induced liver injury. Vet. Human Toxicol., 20, 97-102.

RASOLONIRAINI E. S., 1977. Enzymologie sérique des bovins. Etude bibliographique. Th. Doct. Vét., Toulouse.

RENOULT, E., 1985. De la carence en cuivre et en zinc chez les mammifères. Intérêt du diagnostic analytique. Th. Doct. Vét., Toulouse.

ROBERTS H. E., 1968. Observations on experimental acute fasciolisis in sheep. Br. vet. J., 124, 433-450.

RUSHTON B., 1975. Veterinary laboratory data. BVA Publ. London.

SANDERMAN R. M., HOWELL M. J., 1981. Response of sheep to challenge infection with Fasciola hepatica. Res. vet. Sci, 30, 294-297.

SANDHOLM M., 1974. A preliminary report of a rapid method for the demonstration of abnormal gammaglobulin levels in bovine whole blood. Res. vet. Sci., 17, 32-35.

SATO T., 1984. Application of indocyanine green clearance test in dairy cows. Jpn. J. vet. Sci., 46, 687-692.

SCHMIDL M., 1981. Laboruntersuchungen für die Diagnose und Verlaufskontrolle in der Verterinärmedizin. Boehringer Mannheim GmbH, Mannheim.

SCHMIDT R., SCHMIDT F. W., 1979. Enzyme diagnosis in diseases of the liver and biliary system, 239-292. In SCHMIDT E., SCHMIDT F. W., Advances in clinical enzymology, Karger, Bâle.

SEN M. M., RAHMAN A., MIA A. S., 1976. Liver function tests in goat. Indian vet. J., 53, 504-507.

SHLOSBERG A., BOGIN E., PIPANO E., KLINGER 1., 1973. Serum enzyme levels in experimental theileriosis in calves. Refuah Vet., 30, 24-27.

SHAW F. D., 1974. Sorbitol dehydrogenase in the diagnosis of liver disease of ruminants. Austr. vet. J., 50, 277-278.

SIRAG S. B., CHRISTENSEN N. O., NANSEN P., MONRAD J., FRANDSEN F., 1981. Resistance to Fasciola hepatica in calves harbouring primary patent Schistosoma bovis infections. J. He/mint. 55, 63-70.

SMITH B. L., COE B. D., EMBLING P. P., 1976. Protective effect of zinc sulphate in a natural facial eczema outbreak in dairy cows. N. Z. vet. J., 26, 314-315.

SMITH B. L., EMBLING P. P., TOWERS N. R., WRIGHT D. E., PAYNE E., 1975. The 
protective effect of zinc sulphate in experimental sporidesmin poisoning in sheep intoxication. N.Z. Vet. J., 25, 124-127.

SMITH B. L., STANBRIDGE T. A., EMBLING P. P., 1980. Sheep breed differences in pentobarbitone sleeping-time and response to experimental sporidesmin intoxication. N. Z. Vet. J., 28 , 35-36.

SUTHERLAND R. J., CORDES D. O., CARTHEW G. C., 1979. Ovine white liver disease - an hepatic dysfunction associated with vitamin $\mathrm{B}_{12}$ deficiency. N. Z. vet. J., 27, $227-232$.

SYKES A. R., COOP R. L., ROBINSON H. G., 1980. Chronic subclinical ovine fascioliasis : plasma glutamate dehydrogenase gammaglutamyl transferase and aspartate aminotransferase activities and their significance as diagnostic aids. Res. vet. Sci., 28, 71-75.

TOWERS N. R., 1978. The incidence of sub-clinical facial eczema in selected Waikato dairy herds. N. Z. vet. J., 26, 142-145.

TOWERS N.R., SMITH B. L., 1978. The protective effect of zinc sulphate in experimental sporidesmin intoxication of lactating dairy cows. N. Z. vet J., 26, 199-202.

TOWERS N. R., STRATTON G. C., 1978 . Serum gamma-glutamyl transferase as a measure of sporidesmin-induced liver damage in sheep. N. Z. vet. J., 26, 109-112.

TREACHER R. J., 1972. Galactose elimination as a liver function test in goats. Res. vet. Sci, $13,427-430$.

TREACHER R. J., SANSOM B. F., 1969. Liver function in dairy cows at parturition. Res. vet. Sci, 10. 461-468.

VAN ZYL L. C., 1974. Serum protein fractions as determined by cellulose acetate electrophoresis in Schistosoma Matthei infested sheep. Onderstepoort J. vet. Res., 41, 7-14.

WILLIAMS M. R., MAXWELL, D. A. G., SPOONER R. L., 1975. Quantitative studies on bovine immunoglobulins, normal plasma levels of $\lg _{2}, \lg G_{1}, \lg M$ and $\lg A$. Res. vet. Sci., 18, 314321.

WITTE D. M., 1970. Untersuchungen zur Methodik der Brosulphaleinprobe beim Rind. Inaug. Diss. Hannover.

ZAJICEK D., MAROVA M., KALOUS F., 1972. Changes in serum protein fractions associated with experimental Haemonchus contortus and Trichostrongy/us columbriformis infections in lambs, and the effect of Nilverm (ICI) treatment. Acta vet. Brno, 41, 413-420. 